\title{
Intersections
}

Canadian Journal of Music

Revue canadienne de musique

\section{Eric Salzman et Thomas Desi. 2008. The New Music Theater; Seeing the Voice, Hearing the Body. New York: Oxford University Press. viii, 408 p. ISBN 978-0-19-509936-2 (couverture rigide)}

\section{Martine Rhéaume}

Volume 29, numéro 1, 2009

URI : https://id.erudit.org/iderudit/039115ar

DOI : https://doi.org/10.7202/039115ar

Aller au sommaire du numéro

Éditeur(s)

Canadian University Music Society / Société de musique des universités canadiennes

ISSN

1911-0146 (imprimé)

1918-512X (numérique)

Découvrir la revue

Citer ce compte rendu

Rhéaume, M. (2009). Compte rendu de [Eric Salzman et Thomas Desi. 2008. The New Music Theater; Seeing the Voice, Hearing the Body. New York : Oxford University Press. viii, 408 p. ISBN 978-0-19-509936-2 (couverture rigide)].

Intersections, 29(1), 112-119. https://doi.org/10.7202/039115ar

Tous droits réservés (C) Canadian University Music Society / Société de musique des universités canadiennes, 2009
Ce document est protégé par la loi sur le droit d'auteur. L'utilisation des services d'Érudit (y compris la reproduction) est assujettie à sa politique d'utilisation que vous pouvez consulter en ligne.

https://apropos.erudit.org/fr/usagers/politique-dutilisation/ 


\section{RÉFÉRENCES}

Albèra, Philippe. 2003. "L’opéra ». Musiques. Une Encyclopédie pour le XXI siècle, "1. Musiques du XXe siècle", sous la dir. de Jean-Jacques Nattiez, 377-440. Arles-Paris : Actes Sud/Cité de la musique.

Lacombe, Hervé. 200o. Bizet. Naissance d'une identité créatrice. Paris : Fayard. Lee, Owen. 1999. Une saison à l'opéra, d'Orphée à Ariane. Montréal : Fides. Dahlhaus, Carl. 1989. Nineteenth-Century Music, traduit par J. Bradford Robinson. Berkeley : University of California Press.

Duchesneau, Michel (dir.). 2002. Circuit, musiques contemporaines, "Opéra aujourd'hui ", 12, $\mathrm{n}^{\circ} 2$.

Rio, Marie-Noël et Rostain, Michel. 1988. L’opéra mort ou vif. Paris : Éditions recherches/encres.

Éric Champagne

Eric Salzman et Thomas Desi. 2008. The New Music Theater; Seeing the Voice, Hearing the Body. New York : Oxford University Press. viii, 408 p. ISBN 978-019-509936-2 (couverture rigide).

\section{DÉFINIR UNE FORME D'ART JAMAIS ABOUTIE}

Nouvelle musique de théâtre, nouveau théâtre musical, théâtre de nouvelle musique; il est difficile de traduire en une seule formule le "New Music Theater " dont les compositeurs, chefs d'orchestre, directeurs artistiques et auteurs Eric Salzman et Thomas Desi tentent d'écrire l'histoire pour une toute première fois. Dès les premières lignes de la préface les auteurs écrivent : "L'absence de catégorie et de définition est à la fois la gloire du théâtre musical et son problème $^{1} »$ (p. vii). Un peu plus loin, ils se positionnent comme les défenseurs de cette « forme d'art jamais aboutie ${ }^{2} »$. Les auteurs expliquent cette difficulté en partie par l'origine de l'expression anglaise, calquée de l'allemand Musiktheater qui peut tout autant signifier le lieu où sont jouées les œuvres que les œuvres elles-mêmes. Musicalement, la formule allemande se rapporte plutôt à des œuvres de l'avant-garde, alors que l'équivalent anglais en vient graduellement à désigner une œuvre vocale théâtrale de petite envergure, dont les collaborations Weill/Brecht seraient parmi les premiers exemples (p. 4). Ils affirment que le terme peut désigner, dans ses nombreuses acceptions, aussi bien un opéra traditionnel qu'une comédie musicale, mais ils ajoutent : "Lorsque nous disons nouveau théâtre musical dans ce livre, nous utilisons le terme d'une façon qui se veut presque toujours exclure l'opéra traditionnel, l'opérette et la comédie musicale ${ }^{3}$ " (p. 5). Malgré l'effort déployé dans ces premières pages,

1 « The lack of categorization and lack of definition is at once the glory of music theater and its problem ».

2 "Art form that never happened".

3 "When we say new music theater in this book, we use the term in a way that is almost always meant to exclude traditional opera, operetta, and musicals ». 
c'est l'expérience de la lecture qui compense pour les zones d'ombre de cette définition. Le livre offre une compréhension plus globale du phénomène dont l'histoire est racontée et dont, tout compte fait, le nom importe peu.

Si l'on s'en tient pour l'instant à ces premières pages, on demeure quand même déçu de ce que ces praticiens de l'art musical scénique n’aient pas levé les ambiguïtés qui peuvent exister, par exemple, entre les définitions données ailleurs par divers théoriciens, reproduites plus bas. En effet, si le théâtre musical n'avait jusqu'à présent pas fait l'objet d'une monographie, on lui octroyait néanmoins sa place dans l'histoire de l'opéra du $\mathrm{XX}^{\mathrm{e}}$ siècle. Par exemple, dans la Géographie de l'opéra au XX $X^{e}$ siècle publiée en $2007^{4}$, Hervé Lacombe prend au compte de l'histoire de l'opéra les changements que Salzman et Dési attribuent au théâtre musical, plus particulièrement en ce qui a trait au rôle de l'opéra (ou du théâtre musical) dans la société, à sa durée type (quelques minutes, par opposition aux longues heures chez Wagner) et par les emprunts au jazz et au music-hall (2007, 33-34). La notion de théâtre musical n'est cependant pas écartée de l'ouvrage; Lacombe en reconnaît la souplesse, la liberté de forme et la dissociation des grandes institutions $(2007,21)$.

Pour définir le « Music Theatre " dans le Grove Online, Andrew Clements écrit: « Un terme habituellement utilisé pour caractériser un type d'opéra et de production d'opéra dans lequel le spectacle et l'impact dramatique sont valorisés au détriment de facteurs purement musicaux ${ }^{5} »$. S'il est pratiquement certain, compte tenu de la définition de Salzman et Desi dévoilée dans quelques lignes, que cette compréhension du terme est en désaccord avec celle du livre, et puisqu'il est peu probable que les auteurs n'aient pas consulté le dictionnaire qui la publie, on est alors d'autant plus surpris de ne trouver aucune allusion à cette conception - la bibliographie ne tenant pas compte de l'article -, ne serait-ce que pour la démentir. Dans un chapitre intitulé « Le théâtre musical : métaphore et métonymie ", la définition avancée par Danielle Cohen-Levinas se rapproche des définitions de nos auteurs : "Lorsqu'on parle de théâtre musical, c'est généralement moins pour débattre d'une architecture conforme à ses règles sans loi que pour raviver des antinomies d'un genre, creuset d'idées hostiles à l'institution et d'une institution hostile à l'expérience » $(1994,186)$. C'est, en effet, l'idée d'innovation, hors des maisons d'opéras, qui transparaît dans tout le livre. Cependant, comme c'est d'ailleurs le cas pour l'histoire de la musique du $\mathrm{XX}^{\mathrm{e}}$ siècle, ces hostilités réciproques entre création théâtrale musicale et maisons d'opéras s'estompent avec le passage du temps autant qu'avec celui des pages de ce livre. En effet, le terme "opéra », pourtant méprisé des définitions préliminaires, se voit subtilement réintégré et réhabilité. C'est notamment le cas avec des compositeurs comme le Néerlandais Louis Andriessen (p. 222-223), dont les œuvres sont à cheval sur le théâtre musical et l'opéra, ou encore le Danois Per Nørgård dont l'opéra Nuit des hommes, créé en 1996 dans

4 Recensé dans ce numéro par Éric Champagne.

5 « A term most often used to characterize a kind of opera and opera production in which spectacle and dramatic impact are emphasized over purely musical factors ». 
un théâtre musical, innove dans la façon de véhiculer son message social et dans celle d'amalgamer paroles et musique (p. 224).

Robert Adlington trouve difficile de tracer une ligne séparant le théâtre musical de la musique instrumentale. Il explique ce lien surprenant par un retour à la théâtralité dans l'interprétation, vocale ou instrumentale, d'une musique contemporaine difficile nécessitant un haut degré de virtuosité et, corollairement, d'implication physique $(2005,225-226)$. Si Salzman et Desi n'ont pas emprunté cette avenue pour expliquer l'avènement d'un théâtre musical contemporain, ils n'ont cependant pas laissé de côté le théâtre instrumental (représenté notamment par l'Américain Harry Partch). Et bien que leur définition du théâtre musical suppose la présence obligatoire d'une voix humaine, l'importance de ce critère semble s'estomper au fil des chapitres. Cette situation laisse penser que les auteurs ont dû faire face à quelques difficultés dans la délimitation nominale et heuristique du théâtre musical. Or cet objet d'étude lance finalement un défi à l'étude taxinomique (ou par genre) de la musique du $\mathrm{XX}^{\mathrm{e}}$ siècle.

Dans cette optique, on aurait alors raison d'affirmer qu'il s'agit là d'une forme théâtrale et musicale toujours en pleine transformation, que les auteurs définissent une première fois en page 5 :

Le théâtre musical est un théâtre mû par la musique (c'est-à-dire clairement lié à l'organisation musicale et à son déroulement temporel) où, à tout le moins, la musique, la langue, l'aspect vocal et le mouvement physique existent, interagissent ou sont placés côte à côte dans une sorte d'égalité, mais joué par d'autres interprètes et dans une ambiance sociale différente que les œuvres habituellement classées comme opéras (chantées par des chanteurs d'opéras dans des maisons d'opéra) ou comme comédies musicales (chantées par des chanteurs de théâtre dans des théâtres 'légitimes') ${ }^{6}$.

Il est donc question d'une certaine forme de théâtre expérimental qui n'emploie ni l'émission vocale lyrique opératique ni l'émission vocale puissante et travaillée : plus populaire, la projection est comparable à celle des comédies musicales. De même, elle n'utilise pas non plus les lieux où ces formes de (comment les appeler ?) théâtre musical sont présentées. Ceci dit, cette définition contient les paramètres du théâtre musical - le théâtre, la musique et son lien particulier avec le déroulement temporel, la langue, la vocalisation et le mouvement physique - qui, augmentés de l'interaction avec le public et les particularités nationales des différents théâtres musicaux, forment peu ou prou la structure du livre, que voici plus en détails.

6 «Music theater is theater that is music driven (i.e., decisively linked to musical timing and organization) where, at the very least, music, language, vocalization, and physical movement exist, interact, or stand side by side in some kind of equality but performed by different performers and in a different social ambiance than works normally categorized as operas (performed by opera singers in opera houses) or musicals (performed by theater singers in 'legitimate' theaters) ». 


\section{ORganisation DU LIVRE}

L'appartenance des auteurs à l'univers concret du théâtre musical est établie dès les premières lignes et sert, en quelque sorte, de fil conducteur. D'abord, le livre est lui-même un témoignage du souci de la forme chez les auteurs - on savoure la table des matières dont les titres, directs et humoristiques, laissent présager un traitement du sujet tout en finesse. Les vingt-deux chapitres sont divisés en quatre grandes parties; au gré du sujet, les auteurs se laissent emporter dans de courtes et charmantes digressions, sous la forme de cinq " entr'actes » (en français dans le livre), contenant des anecdotes qui nourrissent la réflexion, mais qui se justifieraient difficilement dans le cadre plus sérieux du reste de l'ouvrage.

Alors que la première partie, intitulée " Music in Music Theater ", et la seconde, "Theater in Music Theater ", traitent de manière historique les thèmes attendus de la voix, du texte et de l'espace scénique, et que la quatrième partie, " After the Show: Taking It Apart ", résume et synthétise les idées du livre tout en leur donnant une certaine ouverture, la troisième section est plus difficile à circonscrire. Portant le titre "Putting It All Together: La Mise en Scène», cette partie couvre près de 200 des 375 pages de contenu du livre et s'occupe, plutôt que de l'art de la mise en scène, alors traité dans la section précédente, de géographies du théâtre musical dans le monde, c'est-à-dire aux États-Unis, en Allemagne, en Italie, en France (ou plutôt en français, le chapitre comprenant une section sur le Québec), enfin dans le nord de l'Europe (chapitres 8 à 12), autant que dans les types de salles, de publics, de médias, de modes d'émission vocale, et ainsi de suite (chapitres 13 à 18). Cela conduit à une forme d'asymétrie dans l'organisation de la matière, comme si les auteurs avaient ici voulu parler de tout ce qui ne pouvait être inséré dans les autres parties.

Dans le domaine du théâtre musical de création, Salzman et Desi sont des érudits - une érudition acquise sur le terrain, par des années de travail dans les maisons de production ou à titre de critique mais aussi par des voyages à travers le monde, la composition et la mise sur pied de telles œuvres ou encore par des réflexions esthétiques. Rappelons à cet effet la longue carrière du compositeur-musicologue qui se cache derrière ce projet, Eric Salzman (né en 1933), auteur de l'excellent Twentieth-Century Music de 1967 quatre fois édité (la dernière en 2001), et d'une expérience semblable de la part du compositeur, metteur en scène et auteur autrichien Thomas Desi (né en 1967). Il résulte de cette expérience un livre aux qualités pédagogiques certaines, surtout dans les deux premières parties, alors que l'histoire de l'opéra remonte à plusieurs reprises à la Camerata Fiorentina et suit les grandes étapes historiques, de la querelle des Bouffons jusqu'au Gesamkunstwerk de Wagner, puis jusqu'à Schoenberg, dans une formule quelque peu répétitive qui s’avère être une stratégie pédagogique efficace : quiconque n'aurait jamais entendu parler de Bayreuth avant d'entamer cette lecture n'est pas perdu lorsqu'il en est question, ce qui rend le livre facile d'approche pour un public plus large que le lectorat musicologique ou musicien. Il n'en est pas autant des quelques termes et noms propres qui relèvent plus exclusivement du théâtre, alors que les Stanislavski, Artaud et autres Brecht sont traités en survol, comme si les auteurs tendaient la main aux 
arts originellement non musicaux du théâtre musical de création à un point tel qu'ils en oubliaient de tendre l'autre à leur lecteur musicien.

Dans la préface, alors que Salzman et Desi soulignent la nouveauté de leur entreprise, ils avertissent le lecteur : "Nous ne sommes ni musicologues, ni historiens de la musique au sens philologique du terme, mais bien praticiens, compositeurs sur le terrain, auteurs et chefs d'orchestre, défenseurs de 'la forme d'art qui n'a jamais abouti'7 »(p. vii). Il s'agit davantage d'une légitimation de leur statut que d'une mise en garde méthodologique; en d'autres termes, ils affirment que leur démarche a une valeur de terrain que n'aurait pas celle d'un musicologue. Mais que l'on abonde ou non dans le sens de cette prémisse, ce livre contient une somme importante d'informations sur des œuvres et des compositeurs dont les travaux n'avaient pas encore été portés par des monographies.

Pour donner une idée de l'abondance des informations contenues dans ce livre, prenons l'exemple du chapitre 11 portant le titre français " Théâtre $\mathrm{Mu}$ sical ». La dernière sous-section du chapitre "Québec » tient en une page et demie. Après avoir mentionné les succès populaires de Starmania et de Notre Dame de Paris, les auteurs s'attardent à Chants Libres et Pauline Vaillancourt et décrivent, en une page à peine, ce groupe dans lequel, selon leur formule, la voix d'un individu est un moteur pour la création (p. 213). Suite à une liste commentée de plusieurs créations de Chants Libres datant de 1992 à 2005, les auteurs traitent de la musique de Claude Vivier et de son opéra Kopernikus en moins de quinze lignes. Offrant des parallèles inédits avec Moïse et Aaron de Schoenberg, Jeanne d'Arc au bûcher d'Honegger et l'Edipus Rex de Stravinski, la description brève est juste, si l'on entend par « Textes en français et en allemand, plus un langage tonal de sa propre invention ${ }^{8} »$ (p. 214, notre italique) les textes en français, en allemand et dans la langue inventée de Vivier, qui est peut-être tonique, mais certes pas tonale. C'est un tour de force que de donner un portrait convaincant de la création musicale théâtrale au Québec en moins de deux pages. Plus encore, la véracité du tableau, combinée à la façon dont les auteurs racontent les aventures opératiques de Ligeti, Pousseur, Cage ou Berio, donnent confiance en leur traitement des nombreux compositeurs étrangers ayant une portée encore locale, dont les travaux nous sont moins connus mais mentionnés en cours d'ouvrage. Mais lorsque les descriptions de compositeurs, de pièces de théâtre musical et de mises en scène se font lassantes, le lecteur comprend que si une partie du livre sert d'outil pédagogique et vise un public assez large, une autre partie remplit quant à elle le rôle d'une chronique, d'un livre de référence préalable à des travaux plus spécifiques. Si ce but n'est pas explicitement énoncé, c'est parce qu'il pourrait sans doute décourager le lecteur; ce dernier se priverait alors d'une manne d'informations brute qu'il lui appartient de compléter.

7 «We are not musicologists, nor music historians in the philological sense, but practitioners, hands-on composers, sometime writers and directors, defenders of "the art form that never happened" ». Nous avons traduit " directors " par « chefs d'orchestre ", puisque les auteurs utilisent le terme «metteur en scène » en français dans leur texte pour faire référence à cette fonction.

8 «French and German texts plus an imaginary tonal language of his own invention ». 
Les auteurs semblent conscients de ne pouvoir aller parfois qu'en surface, aussi offrent-ils à la fin de chaque chapitre une liste plus ou moins longue de références complémentaires que le lecteur est invité à aller consulter. Or ces suggestions de lecture sont si pertinentes (et les différentes langues dans lesquelles ces livres sont écrits ont de quoi donner aux éditeurs de fructueuses idées de traductions) que l'on ne peut que déplorer l'absence de référence de sites Internet : tout d'abord parce que l'avis de professionnels sur les sites dignes de confiance dans leur domaine n'est jamais superflu; et ensuite parce que, toute notion de droits d'auteur mise à part, des extraits vidéos des œuvres les plus expérimentales, décrites de façon parfois laborieuse dans le livre, apporteraient à leur propos et au positionnement historique des œuvres suggérées dans le texte. Cette réserve mise à part, les auteurs et éditeurs ont cependant bien exploité le médium de la monographie imprimée en joignant aux descriptions d'opéras des photos, qui parfois agrémentent le propos - comme la photo de Pauline Vaillancourt incarnant un Dédale robotisé dans The Wings of Daedalus de l'Italien Maurizio Squillante en p. 200 et sur la jaquette du livre -, ou alors complètent judicieusement une description - comme les trois photos reproduites en page 156 et montrant l'aspect laboratoire de Maulwerke - für Artikulationsorgane und Reproduktionsgeräte de l'Allemand Dieter Schnebel.

Au cours de la quatrième partie intitulée "After the Show: Taking it Apart ", les auteurs élaborent une série de questions concernant la nature de la compréhension musicale et la pertinence de l'analyse musicale (p. 310), avant d'en arriver à une position esthétique claire :

Peu importe à quel point l'art peut être dynamique, transitoire ou dépendant du contexte culturel, la beauté peut encore prendre sa place dans la société et devenir un thème plus dominant dans les arts si on le traite correctement comme une abstraction : faire de beaux objets pour aucune autre raison que de créer de beaux objets ${ }^{9}$. (p. 311)

Cette prise de position esthétique est en parfaite cohérence avec les revendications générales qui transparaissent dans le livre. Celles d'une forme d'art actuelle et créative, utilisant toutes les formes d'émissions vocales, amplifiées ou non, et tout nouveau média que l'on croit nécessaire, portée ou non par une intrigue dramatique compréhensible et racontable, mais qui demeure à l'écoute d'un public. Pareille conception, à laquelle on ajoutera la distinction entre l'intégration de nouveaux médias à une œuvre et la création d'œuvres pour justifier l'utilisation de nouveaux médias (p. 368), s'oppose à des schémas flous et mystifiants : que l'on songe, par exemple, à « The music theater object as process or product " (p. 320) auquel il semble manquer une icône et dont les coquilles - plus nombreuses que dans les chapitres précédents - viennent malheureusement brouiller la compréhension de cette section si instructive.

Cependant, l'annexe I réussit le tour de force de résumer l'histoire récente du théâtre musical en un tableau d'une seule page (p. 376). Décrite en trois

9 « However dynamic, transitory, and dependent on cultural context art may be, beauty may again take its place in society and become a more dominant theme in the arts if properly treated as abstraction: beautiful objects made for the sake of creating beautiful objects. ». 
phases - 1900-1930 : caractère expérimental, abstrait et révolutionnaire du modernisme; 1930-1970 : sérialisme et engagement politique; et, 1970-aujourd'hui : (r)évolutions technologiques - cette histoire sans nom propre est claire et concise. Suite à la bibliographie thématique, on trouve à l'annexe III une liste des institutions et festivals présentant du théâtre musical de création aux ÉtatsUnis, au Canada, en Australie et en Europe. Ces annexes viennent à la fois compléter le rôle pédagogique des deux premières parties du livre et le rôle de chronique porté par la troisième.

\section{EN CONCLUSION}

La publication de The New Music Theater est le fruit des recherches d'une vie entière de la part d'Eric Salzman et de Thomas Desi; on y retrouve donc les qualités de synthèse et d'intégration auxquelles Salzman nous a habitués, augmentées d'une connaissance de terrain à travers l'Europe et l'Amérique du nord qui ne saurait être accumulée par un seul individu. Le livre contient une somme considérable d'informations et lance des pistes de réflexion, en plus d'ouvrir les voies de recherches personnelles plus complètes. Il offre une synthèse de ce que l'on sait actuellement du théâtre musical, notamment ses positions esthétiques, décrites avec courage par les auteurs, qui pourraient en guider l'évolution. Les niveaux - histoire, anecdote, prises de position - sont bien équilibrés et rarement mélangés; la distinction entre fait historique et expérience personnelle des auteurs est toujours claire pour le lecteur. Le livre, en sa qualité de première contribution dans le traitement monographique de cette forme d'art, suscitera sans doute des réponses de la part des autres grandes maisons d'édition musicologique. Le lecteur musicologique gagne à cette diversité autant que l'amateur gagne à la pluralité des théâtres musicaux — qu'on les appelle théâtre musical de création, opéra contemporain, opéra traditionnel ou comédie musicale.

\section{RÉFÉRENCES}

Adlington, Robert. 2005. "Music Theatre since the 1960s». Dans The Cambridge Companion to Twentieth-Century Opera, sous la dir. de Mervyn Cooke, 225-243. Cambridge : Cambridge University Press.

Albèra, Philippe. 2003. "L’opéra ". Musiques. Une Encyclopédie pour le XXI siècle, "1. Musiques du XX $\mathrm{X}^{\mathrm{e}}$ siècle ", sous la dir. de Jean-Jacques Nattiez, 377-440. Arles-Paris : Actes Sud/Cité de la musique.

Clements, Andrew. « Music Theatre ». Grove Music Online [consulté le 30 mars 2009].

Cohen-Levinas, Danielle. 1994. " Le théâtre musical : métaphore et métonymie ». Dans Le présent de l'opéra au XX $X^{e}$ siècle; Chemin vers les nouvelles utopies, 184-191. Villeurbanne : Art Édition.

Duchesneau, Michel (dir.). 2002. Circuit, musiques contemporaines, "Opéra aujourd'hui » vol. 12, $\mathrm{n}^{\mathrm{o}} 2$.

Lacombe, Hervé. 2007. Géographie de l'opéra au XXe siècle. Paris : Fayard. 
Salzman, Eric. 1988. Twentieth-Century Music. Englewood Cliffs (NJ) : Prentice Hall. 1ère édition, 1967.

\section{Martine RhÉAume}

Nicolas Dufetel et Malou Haine, éd. 2007. Liszt : un saltimbanque en province. Lyon : Symétrie, xii, 424 p. ISBN 978-2-914373-27-2 (couverture rigide).

Liszt a certainement été l'un des plus célèbres voyageurs du XIX ${ }^{\mathrm{e}}$ siècle. Durant toute sa carrière, il n'a eu de cesse de sillonner l'Europe, changeant régulièrement de domicile et de pays. Deux périodes sont particulièrement saillantes à ce propos : la Glanzperiode des années 1840, au cours de laquelle il ne dispose d'aucun logement permanent et arpente les routes de concert en concert, et la «vie trifurquée » des dernières années de son existence, laquelle lui fait alterner des séjours dans trois villes : Rome, Weimar et Budapest. Le livre recensé ici est consacré aux concerts français du Liszt de la Glanzperiode. Toutefois, le cadre chronologique qui lui est assigné est bien plus large, puisqu'il prend en considération les nombreux concerts donnés par le compositeur depuis son arrivée en France, en 1823. Ces concerts annoncent les circuits de la Glanzperiode, dont l'avènement a été le fruit d'un long cheminement que retrace très pertinemment cet ouvrage.

Ce dernier regroupe les actes du colloque Liszt à Angers organisé par Nicolas Dufetel au Grand Théâtre de cette ville en 2005. S'ajoutent à ces actes d'autres articles sur les concerts de Liszt en "province ". Il convient de souligner d'emblée l'important travail documentaire réalisé dans le cadre de cette étude. Il suffit d'un coup d'œil sur la liste des quelque deux cents concerts donnés par le compositeur en France, en dehors de Paris, entre 1823 et 1846, pour s'en persuader. Ainsi, l'introduction de Nicolas Dufetel et de Malou Haine dresse un inventaire précis des tournées de concerts dans différentes villes de France et celles des pays limitrophes lorsqu'elles constituent une étape de ces tournées (p. 8-17). Rappelons que Nicolas Dufetel est chercheur associé à la Bibliothèque nationale de France (2007/08) et l'auteur d'une thèse de doctorat sur la musique religieuse de Liszt, et que Malou Haine, professeur à l'Université Libre de Bruxelles, a dirigé la collection Perpetuum mobile chez Symétrie au moment de cette publication. À cela s'ajoutent la liste alphabétique d'une soixantaine de lieux de concert, dont la consultation est très aisée (p. 18-19), et la bibliographie (p. 20-22). Ce travail sur les régions françaises vient compléter avantageusement les recherches entreprises par Geraldine Keeling sur les concerts lisztiens à Paris (voir Keeling 1986/1987).

Le livre, intitulé Liszt : un saltimbanque en province, vise à présenter, selon les éditeurs, le pianiste et les "deux aspects souvent opposés de sa personnalité, celui de l'amuseur et celui du créateur» (p. 2). Citant Carl Dahlhaus (1991, 135), les éditeurs conçoivent la virtuosité lisztienne non pas comme une partie " périphérique et épigonale " de la musique, mais bien comme une manière de participer à la " révolution romantique au niveau compositionnel » (p. 2). 